\title{
Suicidio en menores de 26 años en Sevilla
}

\author{
Suicide in people under 26 years of age in Seville
}

\section{Resumen}

La tasa global de suicidios ha sufrido un aumento importante en las últimas décadas, no sólo en los países desarrollados sino a nivel mundial. Es de destacar el especial dramatismo del suicidio en los jóvenes de 15 a 25 años, grupo de población en el que el suicidio es la segunda causa de muerte después de los accidentes de tráfico.

En este artículo se realiza un estudio epidemiológico descriptivo de tipo retrospectivo de las muertes de etiología suicida en menores de 26 años ocurridas en la provincia de Sevilla en el periodo 2007-2010. En él se analizan los factores relacionados con la conducta suicida y se contrastan los resultados con los de la literatura científica existente sobre el tema. La tasa media global de suicidio fue de 1,38 $\pm 0,1$ casos/100.000 habitantes con una relación hombre/mujer de 3:1. La edad media global ha sido de $21 \pm 3$ años ( $21 \pm 3$ años en varones y $20 \pm 4$ años en mujeres). El $22 \%$ de los suicidios ocurre en la capital frente al $78 \%$ registrado en el resto de la provincia. La ahorcadura es el mecanismo más empleado tanto en hombres (71\%) como en mujeres (62\%), seguido de la precipitación. El lugar de comisión del suicidio en ambos sexos es el propio domicilio (72\%). En un 44\% de los casos existían antecedentes psicopatológicos siendo el trastorno depresivo el más frecuente (50\%) seguido de la esquizofrenia paranoide (14\%). El análisis químico-toxicológico fue positivo en el $47 \%$, siendo el alcohol etílico la sustancia más detectada (80\%), bien de forma aislada o combinado con otros tóxicos.

Palabras clave: Suicidio. Incidencia. Sevilla. Autopsia. Medicina forense.

\section{Abstract}

The overall suicide rate has increased significantly in recent decades, not only in developed countries but also worldwide. It is important to underline the prevalence of suicide among young people 15 to 25 years old, a population in which suicide is the second leading cause of death after traffic accidents.

This paper provides a descriptive epidemiological study of confirmed suicides in people less than 26 years of age, which occurred in the province of Seville during the period 2007-2010. The factors associated with suicidal behaviour are presented and compared with those published in the scientific literature on the subject. The average global rate of suicide was $1.38 \pm 0.1$ cases/100.000 inhabitants with a male/female ratio of 3:1. The overall mean age was of $20.9 \pm 3.1$ years ( $21 \pm 3$ years in men and $20 \pm 4$ years in women). Twenty-two per cent of suicides occurred in the capital compared to $78 \%$ in the rest of the province. Hanging was the most widely used method in both men $(71 \%)$ and women (62\%), followed by jumping from height. The most common location to commit suicide in both sexes was at home $(72 \%)$. In $44 \%$ of cases there was a history of psychiatric illness with being depressive disorder the most frequent $(50 \%)$, followed by paranoid schizophrenia (14\%). Toxicological analysis was positive in $47 \%$ cases, with ethanol the most frequently detected chemical $(80 \%)$, either alone or in combination with other toxics.

Key words: Suicide. Rate. Seville. Autopsy. Forensic medicine. Submerged corpse.

\section{F. Cano ${ }^{1}$ \\ A. Rico ${ }^{2}$ \\ R. Marín ${ }^{2}$ \\ M. Blanco ${ }^{2}$ \\ M. Santos ${ }^{2}$ \\ J. Lucena ${ }^{2}$}

${ }^{1}$ Máster en Criminología y Ciencias Forenses. Universidad Pablo de Olavide. Sevilla 2Servicio de Patología Forense. Instituto de Medicina Legal. Sevilla.

Correspondencia: Antonio Rico García Servicio de Patología Forense. IML de Sevilla. C/ M. Romero Martínez, 2 41015 Sevilla E-mail: anricoga@gmail.com

Fecha de recepción: 18.NOV.2011

Fecha de aceptación: 13.JUN.2012 


\section{Introducción}

La conducta suicida en la infancia y adolescencia se ha convertido actualmente en un importante problema de salud por su incremento, su repercusión emocional y por los costes sociales y económicos que comporta ${ }^{1}$. La Organización Mundial de la Salud (OMS) define el suicidio como "un acto con resultado letal, deliberadamente iniciado y realizado por el sujeto, sabiendo o esperando su resultado y a través del cual pretende obtener los cambios deseados"2.

Un reciente artículo de Lancet $^{3}$ pone de manifiesto la magnitud global del problema. La tasa global de suicidios ha sufrido un aumento importante en las últimas décadas, no sólo en los países desarrollados sino a nivel mundial. Es de destacar el especial dramatismo en los jóvenes de 15 a 24 años, grupo de población en que el suicidio es la segunda causa de muerte después de los accidentes de tráfico, independientemente del género ${ }^{4}$.

En España, el suicidio se ha convertido en la primera causa de muerte violenta, desplazando a los accidentes de tráfico y la tendencia es, año a año, lentamente ascendente: en 2008 ya se pasó del "CCC" (corazón, cáncer, carretera) al "CCS" (con "S" de suicidio) ${ }^{5}$.

Según la OMS, a nivel mundial uno de los datos más preocupantes es el aumento de las tasas de suicidio entre los jóvenes entre 15-24 años. Aunque España se sitúa entre las tasas más bajas de Europa, existe una tendencia ascendente mientras que en el resto tiende al descenso o estabilización².

De todo lo anterior se deriva la importancia de conocer las circunstancias que rodean a estas muertes así como los factores de riesgo, necesarios para evaluar el problema y promover una adecuada política de prevención.

En este estudio del suicidio en menores de 26 años en la provincia de Sevilla se han analizado los factores relacionados con la conducta suicida contrastando los resultados con el resto de la literatura científica sobre el tema. También se ha pretendido establecer un punto de partida para posteriores investigaciones, con la finalidad de determinar políticas de prevención efectivas de la conducta suicida tanto en menores de 26 años como en la población general.

\section{Material y métodos}

Se trata de un estudio retrospectivo de suicidios consumados en la provincia de Sevilla durante los años 2007 y 2010 en fallecidos con una edad no superior a los 25 años.
El material utilizado en este trabajo abarca toda la documentación sobre las muertes violentas ocurridas en la provincia de Sevilla durante el período 2007-2010, cuya autopsia fue realizada en el Servicio de Patología Forense del Instituto de Medicina Legal de Sevilla (SPFIMLSE), y cuya etiología suicida había sido establecida y consignada por el médico forense correspondiente.

Las fuentes utilizadas para la realización de este estudio han sido la base de datos de registros del SPFIMLSE, donde está centralizada toda la información de las autopsias de la provincia de Sevilla. En ella se recogen los datos de filiación del cadáver, procedencia, antecedentes patológicos, data de la muerte, causa, mecanismo y etiología de la muerte y resultado de los análisis practicados. Para completar esta información se han consultado los protocolos de levantamiento del cadáver, los informes de autopsia y los resultados de los análisis químico-toxicológicos llevados a cabo en el Departamento de Sevilla del Instituto Nacional de Toxicología y Ciencias Forenses (INTCF). Dichos análisis son realizados a partir de muestras de sangre y orina, determinándose el término positivo en el caso del etanol para concentraciones superiores a 0,1 g/l de sangre, tal y como se establece en los protocolos del INTCF. Concentraciones inferiores a ésta quedan excluidas ya que consideramos podrían tratarse de falsos positivos. En cuanto al resto de sustancias (benzodiacepinas y cocaína), su simple detección en la muestra se considera como positivo, independientemente de su concentración.

Los cálculos de incidencias están realizados según datos de la población real obtenidos del Instituto Nacional de Estadística (I.N.E.), para cada uno de los años (2007 a 2010). Las variables numéricas se resumieron con medias y desviaciones típicas y las variables no numéricas con tablas de porcentajes y frecuencias. Para la comparación entre las distintas variables cualitativas se ha utilizado como prueba no paramétrica el test del Chi-cuadrado. Un valor de $\mathrm{P}$ $<0.05$ es considerado significativo. Para el procesamiento y análisis estadístico de los datos se han utilizado los programas Excel del paquete Microsoft Office y el Programa Estadístico para Ciencias Sociales SPSS 15.0.

\section{Resultados}

\section{Frecuencia y tasas de suicidio}

Durante este periodo se realizaron en el SPFIMLSE un total de 3.609 autopsias, de las cuales 1.855 (51\%) correspondieron a muertes violentas. De estas, 567 (31\%) fueron considerados de etiología suicida, siendo 32 casos (5,6\%) llevados a cabo por menores de 26 años. 
La tasa media global de suicidio en dichos jóvenes entre los años 2007-2010 ha sido de 1,38 \pm 0.1 casos/100.000 habitantes, 2,02 $\pm 0,3$ en el caso de los hombres y 0,71 $\pm 0,1$ en las mujeres (Tabla 1 ).

Sin embargo, si tenemos en cuenta que el primer suicidio registrado tiene lugar a los 14 años y reducimos el margen de edad entre 14 y 25 años, obtenemos una tasa de 2,9 $\pm 1,2$ casos/100.000 habitantes. Observamos pues que esta tasa se duplica lo cual pone de manifiesto que dicha tasa supone casi la mitad de la que se da para la población de Sevilla en su globalidad, y que está en torno a 6 casos/100.000 habitantes y año ${ }^{6}$ (Tabla 2).

\section{Sexo y edad}

Del total de suicidios, 24 fueron cometidos por hombres y 8 por mujeres. Esto se traduce en una tasa media global en los hombres 2,02 $\pm 0,3$ casos/100.000 habitantes frente al 0,71 0,1 casos/100.000 habitantes en las mujeres, lo que representa una relación de 3 hombres por cada mujer.

La edad media global ha sido de $21 \pm 3$ años ( $21 \pm 3$ años en varones y $20 \pm 4$ años en mujeres). La máxima frecuencia se alcanza en los grupos de edad de 20-25 años, con una tasa de incidencia de

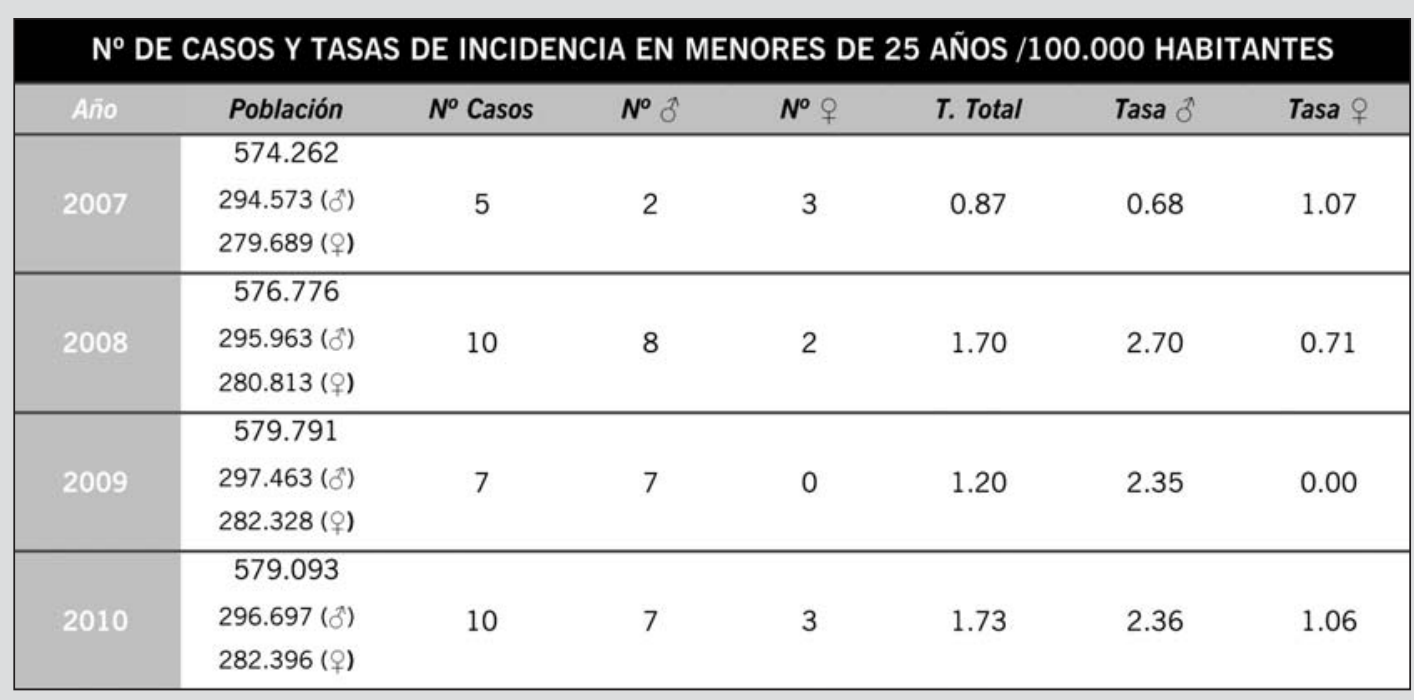

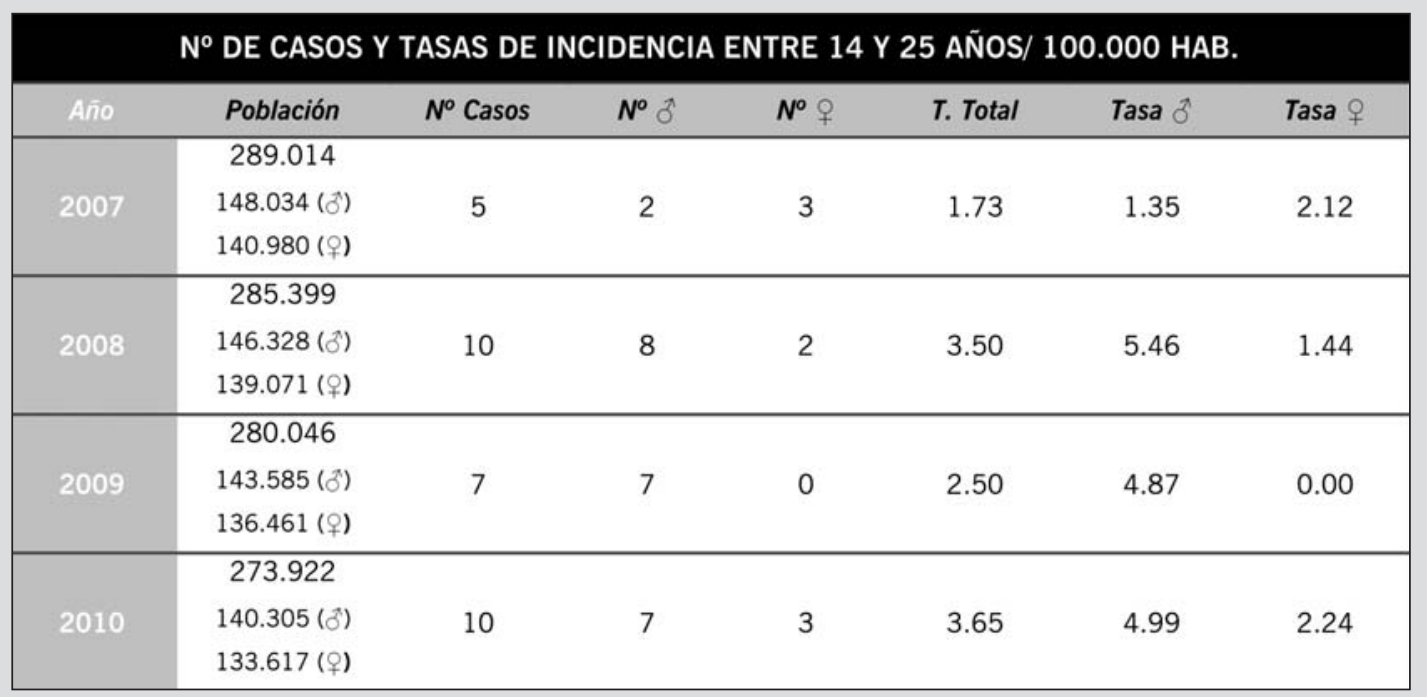

Tabla 1.

Incidencia global población menor de 26 años/100.000 habitantes.

Tabla 2.

ncidencia global población 14-25 años/100.000 habitantes. 
4,5 casos/100.000 habitantes frente a los 0,5 casos/100.000 habitantes en el grupo de edad de 0-19 años. Por tanto, este es el grupo etario de mayor riesgo disminuyendo de forma significativa en la edad infantil y permaneciendo estable en el grupo de 14 a 20 años (rango 0-3 casos) (Figura 1).

\section{Distribución temporal}

Para el análisis de la hora, sólo han sido considerados 31 de los 32 casos estudiados, ya que uno de ellos fue hallado en avanzado estado de descomposición, resultando por tanto muy difícil determinar con exactitud la data de la muerte. Dividiendo el día en cuatro intervalos horarios, se obtiene una mayor incidencia $(29,0 \%)$ entre las 00.01 y las $06.00 \mathrm{~h}$ de la madrugada. Respecto al día de la semana, el $62,5 \%$ de los suicidios se comenten en día laborable mientras que el $37,5 \%$ ocurren en fin de semana
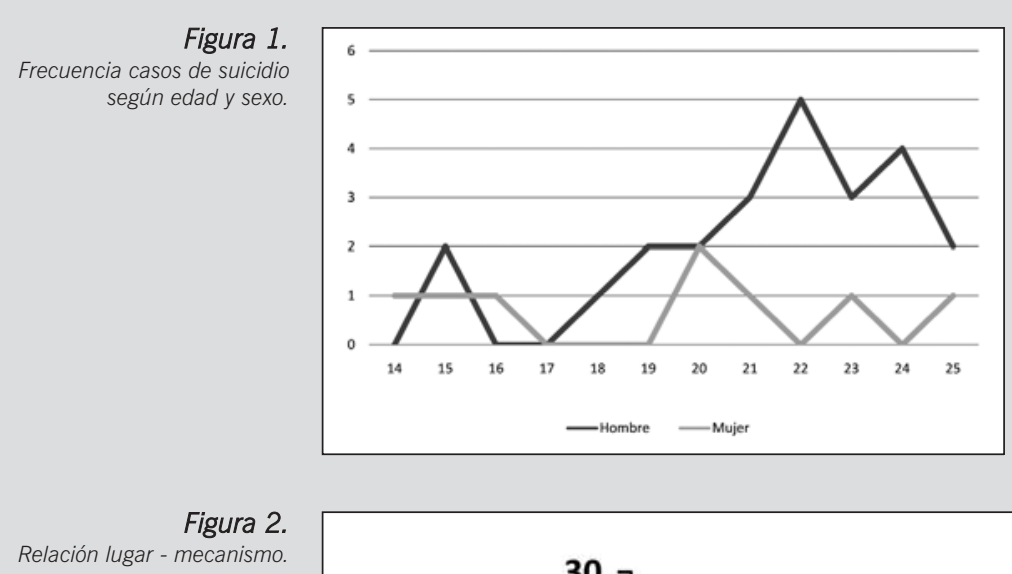

\section{Mecanismo}

El mecanismo más frecuentemente empleado fue la ahorcadura (69\%), seguido de la precipitación (19\%) $(P<0,05)$. Otros procedimientos, aunque en menor medida, fueron las armas de fuego (9\%) y la sumersión (3\%).

Si relacionamos el mecanismo con el sexo, tanto en hombres como en mujeres el mecanismo más comúnmente empleado es la ahorcadura seguido de 
la precipitación. Los varones utilizan exclusivamente las armas de fuego y, con menor frecuencia, la sumersión (Figura 3).

\section{Antecedentes psicopatológicos}

Estos antecedentes se han determinado en primer lugar a partir de la anamnesis realizada a los familiares de cada fallecido, y posteriormente contrastándose y verificándose tal información ya que en todos los casos se trataba de psicopatologías ya diagnosticadas.

El 44\% (14 casos) de los sujetos tenía antecedentes psicopatológicos, siendo el trastorno depresivo la patología más frecuentemente diagnosticada (50\%), seguido de la esquizofrenia paranoide (14\%), trastorno de personalidad (7\%), trastorno bipolar (7\%) y trastornos de conducta (7\%). El 14\% de los casos restantes corresponde a toxicomanías. En un 56\% (18 casos) no se conocían antecedentes psicopatológicos.

\section{Análisis químico-toxicológico}

Se tomaron muestras de sangre y orina en todos los casos para la realización de un análisis químico-toxicológico de etanol, psicofármacos y drogas de abuso. Los resultados fueron negativos en 17 (53\%) y positivos (se detecta una o varias de estas sustancias a diferentes concentraciones) en los 15 casos restantes (47\%). En el grupo donde los resultados fueron positivos, el etanol se detectó en un total de 12 casos (80\%), de forma aislada en el $58 \%$ (7 casos), combinado con benzodiacepinas en el $25 \%$ (3 casos) y combinado con benzodiacepinas y cocaína en el $17 \%$ ( 2 casos). En 3 casos (20\%) se detectaron únicamente benzodiacepinas (Figura 4).
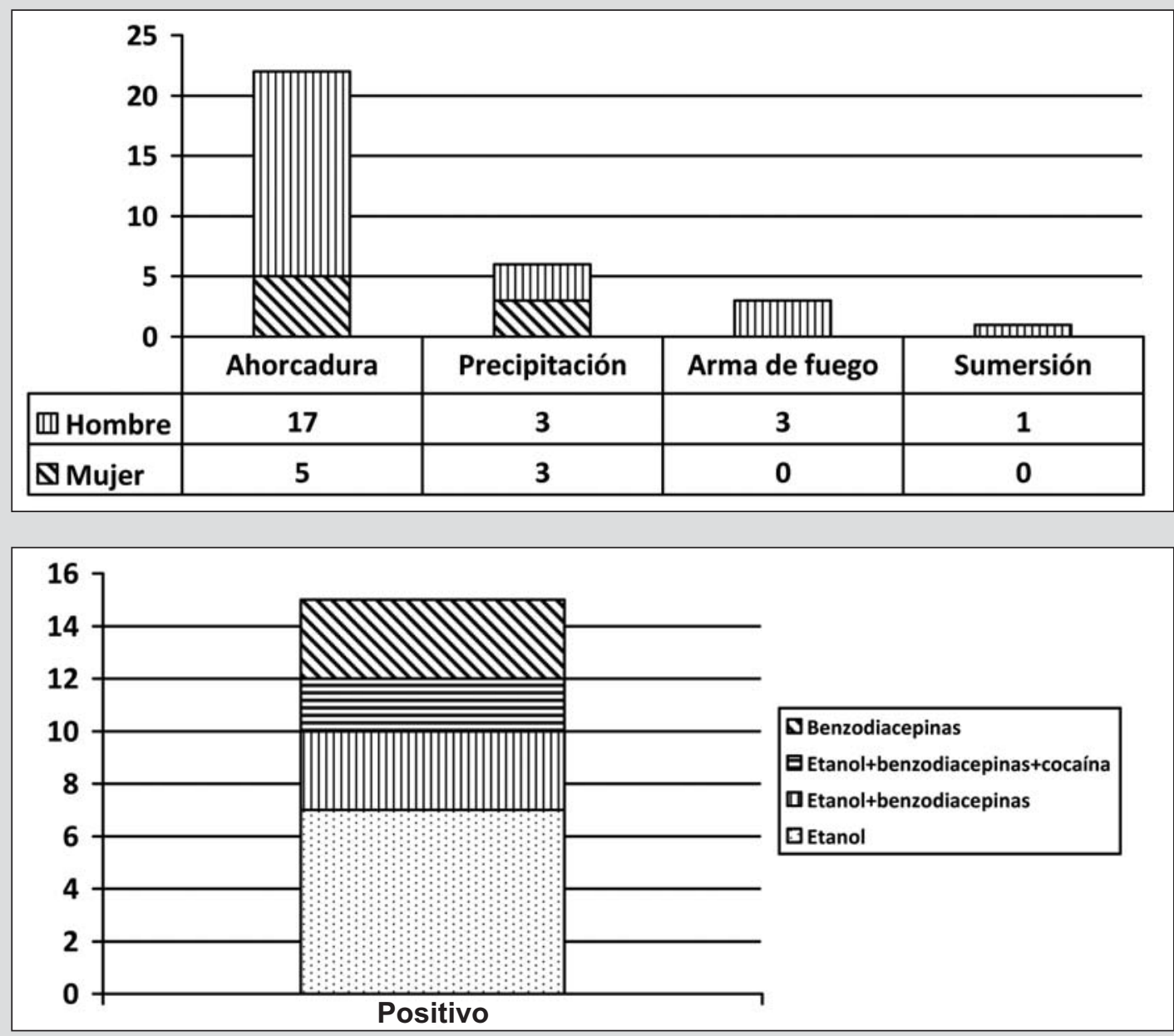

Figura 3.

Comparación mecanismos por sexo.

Figura 4.

Resultados toxicológicos. 


\begin{tabular}{|c|c|c|c|c|c|c|c|c|c|}
\hline \multirow{3}{*}{$\begin{array}{r}\text { Tabla } 3 . \\
\text { Análisis toxicológico. } \\
\text { Concentración en } \\
\text { sangre de sustancias. }\end{array}$} & \multicolumn{3}{|c|}{ ETANOL } & \multicolumn{3}{|c|}{ BENZODIACEPINAS } & \multicolumn{3}{|c|}{ COCAIINA } \\
\hline & Mínima & Máxima & Media & Mínima & Máxima & Media & Mínima & Máxima & Media \\
\hline & $0.12 \mathrm{~g} / \mathrm{L}$ & $2.80 \mathrm{~g} / \mathrm{L}$ & $0.99 \mathrm{~g} / \mathrm{L}$ & $0.03 \mathrm{mg} / \mathrm{L}$ & $0.46 \mathrm{mg} / \mathrm{L}$ & $0.32 \mathrm{mg} / \mathrm{L}$ & $0.09 \mathrm{mg} / \mathrm{L}$ & $1.98 \mathrm{mg} / \mathrm{L}$ & $0.59 \mathrm{mg} / \mathrm{L}$ \\
\hline
\end{tabular}

Respecto a las concentraciones de cada una de las sustancias, los datos quedan reflejados en la Tabla 3.

\section{Discusión}

El suicidio en jóvenes es una realidad cada vez más patente en nuestro país. Es una tragedia que se menciona pocas veces en nuestra sociedad, suele callarse y evadirse pues nos enfrenta directamente con la realidad de que los jóvenes de forma consciente se causan la muerte. Es un tema que impacta y cuestiona nuestro sistema familiar y social. Sin embargo, es importante encarar el problema y tratar de comprender las posibles razones para que un adolescente, acabe repentinamente con su existencia. Un mayor conocimiento sobre los factores de riesgo y de protección del suicidio en la adolescencia son la base empírica para la prevención ${ }^{7}$ ya que puede promover las primeras intervenciones específicas, lo cual reduciría el riesgo futuro de la conducta suicida ${ }^{8}$.

En el cuatrienio 2007-2010 se investigaron en el SPFIMLSE 567 muertes suicidas de las que 32 fueron llevadas a cabo por jóvenes menores de 26 años. Si tenemos en cuenta que todos los casos tienen lugar entre los 14 y 25 años, la tasa de suicidio es de 2,9 $\pm 1,2$ casos/100.000 habitantes, cifras más que alarmantes si la comparamos con la tasa media nacional de suicidio general que para España en su globalidad viene a cifrarse en torno a los 5 suicidios por cada 100.000 habitantes y año6.

En cuanto a la ratio inter-sexos, se estima que el suicidio continúa siendo más frecuente en hombres que en mujeres, siendo la relación de 3:1. Estos resultados coinciden con los obtenidos en otros estudios nacionales ${ }^{4,9}$. Por último señalar que en España el sexo debe considerarse un factor diferencial puesto que las tasas de suicidio llegan a ser hasta tres veces más altas en varones que en mujeres en todos los grupos de edad.

La media global de edad fue de $21 \pm 3$ años, en hombres $21 \pm 3$ años y en mujeres $20 \pm 4$ años. La má- xima frecuencia de casos en hombres tiene lugar entre los $20-25$ años con el $79 \%$ de los suicidios. En el caso de las mujeres no se da un periodo de máxima frecuencia. Este dato también coincide con la mayoría de las investigaciones donde el riesgo se incrementa con la edad, siendo extremadamente raros los casos de suicidio consumado en menores de 12 años, resultando más frecuente para este tramo de edad las conductas parasuicidas ${ }^{10}$.

A diferencia de otros estudios ${ }^{9}$, si hemos observado diferencias en cuanto a la fecha de comisión. El mayor número de suicidios se ha producido en la primera quincena de los meses de abril, mayo y julio, con prácticamente la mitad de los casos registrados a lo largo de todo el año. No se observan diferencias llamativas en la distribución de los casos de acuerdo con la hora del suceso. Ambos sexos optan por cometer el suicidio durante las horas nocturnas (9 casos entre las 00.01 y las $06.00 \mathrm{~h}$ ) seguido del mediodía (8 casos), aunque no existe una gran diferencia con la incidencia de suicidio registrada a otras horas del día. La primavera y el verano son las estaciones con mayor incidencia de suicidios. Estos datos también se repiten en las estadísticas obtenidas en otros estudios ${ }^{10,11}$. En cuanto a los días de la semana los dos sexos coinciden ya que tanto hombres como mujeres se suicidan más durante el fin de semana (sábados y domingos), aunque esta diferencia no es significativa.

Tras el análisis de los datos según el municipio en que tuvo lugar el suicidio, hemos encontrado un porcentaje diferencial considerable entre los suicidios en Sevilla capital (22\%) y en el resto de la provincia (78\%). No obstante, hay que ser cautos para sacar conclusiones definitivas al no disponer de las cifras de población para el rango de edad estudiado, lo que ha impedido obtener las tasas correspondientes. En nuestra opinión, esta diferencia confirmaría la hipótesis planteada en otros estudios según la cual la incidencia de suicidio es mayor en los medios rurales que en los urbanos, permitiendo establecer una correlación entre el suicidio y el tipo de entorno geográfico ${ }^{12}$.

El domicilio sigue siendo el lugar de preferencia para la consumación del acto suicida, tanto en hombres 
como en mujeres en el $72 \%$ de los casos. Esto coincide con los datos obtenidos en la mayoría de estudios similares como los llevados a cabo en Sevilla $(48 \%)^{11}$, Cambados $(61 \%)^{13}$ o Badajoz $(57 \%)^{14}$, aunque difiere en el caso de las mujeres de otros como el realizado en Alicante en $2005^{4}$ y según el cual ellas prefieren consumar los hechos en la vía pública mediante precipitación.

En cuanto a los mecanismos empleados para cometer el suicidio, el más utilizado tanto por hombres como por mujeres es la ahorcadura. Esto indica un cambio de tendencia en los mecanismos usados por el sexo femenino ya que, según algunos estudios ${ }^{4,11}$, el más empleado era la precipitación y la ingesta de tóxicos. Actualmente la ahorcadura ocupa el primer lugar como mecanismo de suicidio para mujeres, seguido de la precipitación. En el caso de los hombres, la ahorcadura ya ocupaba el primer lugar como mecanismo más empleado en $2004^{11}$. Un dato importante a señalar es que el uso de arma de fuego es exclusivo del sexo masculino.

Durante muchos siglos se ha tendido a interpretar el suicidio como una manifestación sintomática de enfermedad mental, especialmente de los trastornos depresivos y de la esquizofrenia ${ }^{11}$. Según las teorías de Delmas, todos los suicidios eran patológicos, no siendo otra cosa que una manifestación más de la enfermedad mental ${ }^{6}$. Por ello se ha considerado importante incluir este tipo de antecedentes personales como variable de estudio, a fin de aproximarnos a la influencia real que las enfermedades mentales pueden tener en el desencadenamiento de los actos suicidas.

En Sevilla el 44\% de los sujetos tenía antecedentes psicopatológicos, datos que no están en la línea de otras investigaciones sobre el suicidio consumado que señalan que solo un bajo porcentaje estaría libre de un diagnóstico psicopatológico ${ }^{10}$. Probablemente, en nuestra serie había un porcentaje de casos aún no diagnosticados o probablemente larvados.

Respecto al tipo de patología, se confirma que los trastornos del estado de ánimo son los más frecuentes ${ }^{10}$.

Con respecto al resultado de los análisis toxicológicos, el etanol fue la sustancia más detectada (80\%) en los casos donde dichos resultados fueron positivos ( 15 casos, el 47\%), seguido de las benzodiacepinas y cocaína. Estos datos coinciden con los obtenidos en un estudio realizado en Cambados $^{13}$ y en Alaska ${ }^{7}$, en los que el etanol fue la sustancia más detectada (50\% de los casos). Diversas investigaciones han analizado el vínculo entre el alcohol y la conducta suicida, que parece ser más fuerte en los grupos más jóvenes que en los de mayor edad. La mayoría de los autores se han centrado en la hipótesis del alcohol como precursor del comportamiento suicida, debido a los problemas que causa en la cognición y el afecto (alteración del juicio y del estado de ánimo, aumento de la impulsividad, etc.). En base a los resultados obtenidos, podría decirse que la severidad de la ideación suicida parece aumentar con el consumo de alcohol, en un aparente intento de regular o escapar de una situación negativa ${ }^{15}$.

\section{Limitaciones}

Debemos resaltar la ausencia de algunas variables que hubieran aportado, probablemente, datos de gran interés respecto al fenómeno estudiado, como son: ocupación laboral, nivel socio-cultural, estructura o funcionamiento familiar, estado civil de los padres, antecedentes psiquiátricos en la familia, existencia de nota suicida, etc. Pero desafortunadamente en esta ocasión, no ha sido posible acceder a dichas variables a partir de la base de datos. Consideramos también que el hecho de no haber podido contrastar los resultados obtenidos con un grupo control es otra limitación a tener en cuenta para posteriores estudios. Finalmente, el número de suicidios analizados es escaso por lo que sería necesario confirmar estos resultados con una muestra más amplia.

\section{Conclusiones}

El suicidio de menores de 26 años en Sevilla tiene una incidencia baja si la comparamos con la media de España. No obstante, el problema es completamente diferente si consideramos el suicidio entre 14 y 25 años cuya tasa de incidencia fue de 2,9 $9 \pm 1,2$ casos/100.000 habitantes y año.

Es de destacar que un $44 \%$ de los sujetos tenía antecedentes psicopatológicos, siendo la depresión el trastorno registrado con más frecuencia seguido de la esquizofrenia paranoide. En los casos con análisis toxicológico positivo, el etanol fue el tóxico más frecuentemente encontrado. Ambas variables son susceptibles de modificación en relación a la prevención de las conductas suicidas.

Muy relevante nos parece el hecho de que el $78 \%$ de los casos tuvieron lugar fuera de la capital, en áreas no urbanas o rurales, debiendo incidir en que son probablemente estas zonas las más necesitadas de detección de posibles conductas suicidas en jóvenes y donde deben fomentarse con más interés las campañas socio-sanitarias de prevención. 


\section{Agradecimientos}

Al Dr. P. James Macaluso por la revisión crítica del texto y la traducción al inglés. A la Dra. Ana Fernández
Palacín por la ayuda en el análisis estadístico. A los revisores por su extraordinario trabajo que ha permitido mejorar sustancialmente la versión original del manuscrito.

\section{Bibliografía}

1. Pérez García J, Del Pino Montesinos JI, Ortega Beviá F. El Adolescente, la muerte y el suicidio. Jornadas Europeas para la Prevención de la Violencia en el Medio Escolar. Alicante, Mayo 2002. Disponible en: http://www. bvsde. paho.org/bvsacd/cd90/1604PERado.pdf. Consultado el 10.11.11.

2. Grupo de Trabajo de la Guía de Práctica Clínica sobre la Depresión Mayor en la Infancia y en la Adolescencia. Guía de Práctica Clínica sobre la Depresión Mayor en la Infancia y en la Adolescencia. Plan de Calidad para el Sistema Nacional de Salud del Ministerio de Sanidad y Política Social. Axencia de Avalacion de Tecnoloxias Sanitarias de Galicia (avalia-t); 2009. Guías de Práctica Clínica en el SNS.: avalia-t No 2007/09.

3. Hawton K, Van Heeringen K. Suicide. Lancet 2009;373:1372-81.

4. Ayala G, Martí JB, Rodés F. Incidencia del suicidio consumado en el partido judicial de San Vicente del Raspeig (Alicante). Cuad Med Forense 2005; 11:119-29.

5. Instituto Nacional de Estadística (I.N.E.). http://www.ine.es/.

6. Romero JL, Gamero JJ. El suicidio consumado en el partido judicial de Huelva. Cuad Med Forense 2005; 11:5-9.

7. Perkins R, Sanddal TL, Howell M, Sanddal ND, Berman A. Epidemiological and follow -back stud y of suicides in Alaska. Int J Circumpolar Health 2009;68:3.
8. Cash SJ, Bridge JA. Epidemiology of youth suicide and suicidal behavior. Curr Opin Pediatr 2009; 21:613-9.

9. Gutiérrez JM, Molina FJ. El suicidio consumado en Murcia, 1990-1992. Anales de Psicología 1996;12:207-15.

10. Venceslá JF, Martínez JA. Conducta autolítica y parasuicida. Características sociodemográficas en población infantojuvenil de ámbito rural. Rev Asoc Esp Neuropsiq 2002;84:49-64.

11. Miguel L, Lucena J, Heredia F, Rico A, et al. Estudio epidemiológico del suicidio en Sevilla en 2004. Cuad Med Forense 2005;11:43-53.

12. Organización Mundial de la Salud (O.M.S.). Informe Mundial sobre la Violencia y la Salud. 2002. Disponible en: http://www.who.int/violence injury prevention/violence/world_report/en/summary_es.pdf. Consultado el 3.11.11.

13. Dorado E, Rodés F. Suicidio por ahorcadura en el partido judicial de Cambados (1989-1998). Estudio epidemiológico y comparativo. Cuad Med Forense 2000;19:9-19.

14. Casado M, Sánchez F, Hernández ML, Merino MJ. Análisis del suicidio consumado en el Partido Judicial de Badajoz durante el periodo 1990-1995. Cuad Med Forense 1998;13:77-83.

15. González VM, Bradizza CM, Collins RL. Drinking to Cope as a Statistical Mediator in the Relationship Between Suicidal Ideation and Alcohol Outcomes Among Underage College Drinkers. Psychol Addict Behav 2009; 23: 443-51. 